\title{
Optimization of Enzymatic Extraction Barley Polysaccharides Orthogonal Test Method
}

\author{
Yong-guang $\mathrm{Bi}^{1,2, \star}$, Yu-min $\mathrm{Li}^{2}$ \\ ${ }^{1}$ Guangxi Key Laboratory of Petrochemical Resource Processing and Process Intensification Technology, Guangxi University, Nanning \\ 530004, China \\ ${ }^{2}$ School of Pharmacy, Guangdong Pharmaceutical University, Guangzhou 510006, Guangdong, China
}

\begin{abstract}
In order to optimize Enzymatic Extraction barley polysaccharides parameters, the use of orthogonal test to optimize their processes, affecting the cellulase barley polysaccharide extracted five factors, the maximum temperature hydrolysis, the solid-liquid ratio on minimum. The impact of the extraction rate of polysaccharides barley The order has to: hydrolysis temperature $>\mathrm{PH}$ value $>$ hydrolysis time $>$ enzyme concentration $>$ solid-liquid ratio, based on the experimental parameters, in order to obtain a higher extraction rate, optimization of process parameters of various factors hydrolysis temperature is $55{ }^{\circ} \mathrm{C}$, hydrolysis time $120 \mathrm{~min}$, solid-liquid ratio of 1:30, the amount of enzyme $0.020 \mathrm{~g}$, $\mathrm{PH}$ value 4 , in this condition of polysaccharide extraction rate $2.23 \%$.
\end{abstract}

Barley as grasses, are annual or perennial herb. Fall harvest mature plants cut, dried, lay the fruit, and then dried, remove the shell, brown seed coat and impurities, collected seeds, which we often see in everyday barley. Barley cultivation in our long history, is one of the oldest food and medicine are good grain species. Most of the country are produced in Fujian, Hebei and other provinces based. Because of the high nutritional value of barley, so as the "king of the world grasses" and the "life and health of Wo." Japan has recently been listed as anti-cancer foods, and therefore worth double.

Barley have easy digestion and absorption characteristics, is commonly used in traditional Chinese medicine, it is generally eat food. Whether it is used for medical or tonic, the role is very relaxed. Pale barley and sweet, slightly cold, favorable water swelling, spleen dewetting, getting in addition to weakness, heat pus and other effects, for the common benefits of water Shenshi medicine. Meanwhile, the barley is a kind of beauty food, eat it can keep the skin shiny and delicate, serve to eliminate acne, spot birds, age spots, pregnancy spots, melasma, the effect of scaling, acne, chapped, rough skin, also have a good effect.

Cellulases ( $\beta-1,4-$ glucan -4- glucanohydrolase) are generated to degrade cellulose glucose general term for a group of enzymes, it is not a single enzyme, but a synergistic effect of the multicomponent enzymes, an enzyme, exo- $\beta$ - mainly by glucanase, endo- $\beta$ - glucanase and $\beta$ - glucosidase enzyme composition, there is a high dynamic xylanase. Acting on the product derived from cellulose and cellulose out. Microbial cellulase has a very important role in the transformation of insoluble cellulose into glucose and destruction of cell walls in terms of fruit and vegetable juices to increase juice yield and so on.

Cellulase preparation itself is a kind of protease, amylase, pectinase and cellulase by multi enzyme complexes. In this multi enzyme complex with the product of an enzyme can serve as a substrate for another enzyme, and thus smoothly within the gastrointestinal digestion. That is to say, cellulose, in addition to the direct degradation of cellulose, to promote the decomposition of the low molecular compounds that are easily digested and absorbed by the animal, but also with other enzymes to improve the decomposition and digestion of dairy cow feed nutrients.

In the process of improving the decomposition of cellulose and semi cellulose, it can promote the dissolution of the plant cell wall, which can be easily digested by the large molecular polysaccharide, protein and lipid. Cellulase preparation can activate the secretion of endogenous enzymes, complement the lack of endogenous enzymes, and adjust the endogenous enzymes to ensure the normal digestion and absorption function of animals, play a role in preventing disease and promoting growth. Elimination of anti - nutritional factors, promote the growth of low molecular weight compounds, but also with other enzymes to improve the decomposition and digestion of dairy cow feed nutrients.

In alcohol fermentation of cellulase can significantly improve the alcohol and liquor liquor yield and raw material utilization ratio, reduce solution viscosity, shorten the fermentation time and wine taste mellow, fusel oil content was low. There are two reasons to increase the yield of alcohol: one is that some of the raw materials can be decomposed into glucose for the use of glucose. The application of cellulase in malt beer industry production can increase the solubility of wheat and accelerate germination, reduce the content of a single glucose saccharifying liquid, improve the performance of the filter, is conducive to the alcohol distillation. Cellulase treatment beer lees can improve beer lees enzymolysis rate of more than $10 \%$; application of cellulase in Fen flavor liquor quality and liquor yield can be increased by $13 \%$, and do not affect the sensory quality of the wine; in sake production in Japan, dip meters adding $0.02 \%$ $0.1 \%$ cellulase immersion $17 \mathrm{~h}$, rice with good solubility, saccharification and fermentation smoothly, rosacea, high liquor yield.

Adding cellulase in soy sauce brewing process, expansion softening damage to the cell membrane of soybean raw material, the inclusion in the cell protein and carbohydrates release, this can improve soy sauce concentration and improving the quality of soy sauce, and can shorten the production cycle, improve productivity, and the major indexes were increased by $3 \%$. The solid koji, solid fermentation and solid-state acetic acid 
fermentation production process on the application of cellulase in brewing vinegar were system research.

Therefore, this study on the basis of preliminary studies on the use of Orthogonal Design cellulase extracted barley polysaccharides parameters, with a view to large-scale industrial applications polysaccharide extracted barley provide a theoretical basis and reference.

Cellulose is widely used in nature. Bacteria, fungi, animals, etc., can produce cellulase. Commonly used in the production of cellulase from the fungus, more typical of the genus(Trichoderma), Aspergillus (Aspergillus) and Penicillium.The production of cellulase is easy to degenerate, resulting in reduced production capacity.

Bacteria producing cellulase yield less, endoglucanase, most of crystalline cellulose no degradation activity and produced enzymes are intracellular enzymes or adsorbed on the cell wall, not secreted into the culture liquid and increases the difficulty of extraction and purification, so the bacteria research study less. But the cellulase produced by bacteria is generally the most suitable $\mathrm{pH}$ for neutral to alkaline. In recent 20 years, with the successful application of neutral cellulase and alkaline cellulase in cotton fabric washing process and detergent industry, bacterial cellulase preparation has been shown to have good application prospect.

Cellulase has been widely used in food industry and environmental industry. In alcohol fermentation, cellulase added use can increase the rate of raw materials, and improve the quality of liquor.

China is a country with a very tight feed resource, with little land and a large population. To maintain the sustainable development of China's feed industry and animal husbandry, it is necessary to solve the feed problem, otherwise it will seriously restrict its development. Cellulose is a very rich resource in nature, and it is 800-1200 glucose molecules. Therefore, through microbial fermentation to make full use of waste of agricultural and sideline products, straw, chaff, the production of cellulase additive, used to improve the production performance of livestock and poultry, improve the feed utilization rate, improve the nutritional value of feed, reduce the feed cost and increase economic benefits, has a broad development prospect. In the future, we should further strengthen the research and development :(1) to further strengthen the mechanism of the action of cellulase: cellulase used in feed, the role of animal digestive tract, the mechanism is not clear. In theory, it is difficult to determine the amount of the added amount, the impact of many factors, the effect is not ideal. The optimum addition amount of cellulase for single use of various raw materials is also studied, which will severely restrict the application of cellulase.(2) the production and activity of enzymes are not high and the cost is high. The research work of strain breeding and fermentation technology should be strengthened in the future to increase its yield and activity. In particular, we should pay attention to the application of DNA gene recombination technology to produce high activity and high yield.(3) to strengthen the detection of cellulase: Although the detection method of cellulase, but can be suitable for the detection of feed is not, which gives the practical application of the product quality, determine the amount of cellulase added is also very difficult, should organize the relevant forces to develop a unified testing method for production.f cellulase.

\section{Materials and methods ${ }^{[2]}$}

\subsection{Raw materials and reagents}

Coix chinensis Tod. (Origin: Guizhou, specifications: $0.5 \mathrm{~kg} / \mathrm{bag}$, Hubei Jingui Chinese Herbal Medicine Co., Ltd.). After sifted through a 40 mesh sieve to obtain honeysuckle powder and set aside. Glucose reference, Chinese medicines and biological products; phenol, concentrated sulfuric acid and deionized water, were of analytical grade.

\subsection{Instruments and Equipment}

H-6 constant temperature water bath (Jintan Honghua Instrument Factory); RE-52CS rotary evaporator (Gongyi City, the British valley to China Instrument Factory); I CHINABRAND circulating water pump (Gongyi City, the British valley to China instrument); UV1101 UV / Vis spectrophotometer (Shanghai Tian Mei scientific Instrument Co.); AY120 electronic analytical balance (Shimadzu Corporation).

\section{Results and analysis}

\subsection{Orthogonal}

Orthogonal experiment amount of barley each experiment is $2.000 \mathrm{~g}$. Enzyme extraction temperature, extraction time, solid-liquid ratio, the amount of enzyme, $\mathrm{PH}$ value factors, each set four levels of design (see Tab.1), examine cellulase extracted barley polysaccharides, results shown in Tab.2, Tab.3.

Tab.1 Orthogonal head design

\begin{tabular}{cccccc}
\hline & \multicolumn{5}{c}{ Factor } \\
\hline Level & Temperature $/{ }^{\circ} \mathrm{C}(\mathrm{A})$ & Time/min & solid-liquid ratio & enzyme/amount & PH \\
& & $(\mathrm{B})$ & $\mathrm{g}: \mathrm{mL}(\mathrm{C})$ & $\mathrm{g}(\mathrm{D})$ & Value(E) \\
1 & 30 & 30 & $1: 10$ & 0.004 & 3 \\
2 & 40 & 60 & $1: 20$ & 0.012 & 4 \\
3 & 50 & 120 & $1: 30$ & 0.016 & 6 \\
4 & 55 & 150 & $1: 40$ & 0.020 & 8 \\
\hline
\end{tabular}


Tab.2 Orthogonal test results

\begin{tabular}{ccccccc}
\hline Test No. & $\mathrm{A}$ & $\mathrm{B}$ & $\mathrm{C}$ & $\mathrm{D}$ & $\mathrm{E}$ & Extraction rate $/ \%$ \\
\hline 1 & $1(30)$ & $1(30)$ & $1(1: 10)$ & $1(0.004)$ & $1(3)$ & 1.47 \\
2 & 1 & $2(60)$ & $2(1: 20)$ & $2(0.012)$ & $2(4)$ & 1.81 \\
3 & 1 & $3(120)$ & $3(1: 30)$ & $3(0.016)$ & $3(6)$ & 1.71 \\
4 & 1 & $4(150)$ & $4(1: 40)$ & $4(0.020)$ & $4(8)$ & 1.59 \\
5 & $2(40)$ & 1 & 2 & 3 & 4 & 1.48 \\
6 & 2 & 2 & 1 & 4 & 3 & 1.89 \\
7 & 2 & 3 & 4 & 1 & 2 & 1.78 \\
8 & 2 & 4 & 3 & 2 & 1 & 2.02 \\
9 & $3(50)$ & 1 & 3 & 4 & 2 & 1.80 \\
10 & 3 & 2 & 4 & 3 & 1 & 1.93 \\
11 & 3 & 3 & 1 & 2 & 4 & 2.06 \\
12 & 3 & 4 & 2 & 1 & 3 & 2.05 \\
13 & $4(55)$ & 1 & 4 & 2 & 3 & 2.20 \\
14 & 4 & 2 & 3 & 1 & 4 & \\
15 & 4 & 3 & 2 & 4 & 1 & \\
16 & 4 & 4 & 1 & 3 & 2 & \\
K1 & 6.60 & 7.04 & 7.44 & 7.36 & 7.24 & \\
K2 & 6.96 & 7.48 & 7.40 & 7.60 & 7.92 & \\
K3 & 7.72 & 7.72 & 7.56 & 7.20 & 7.52 & \\
K4 & 8.48 & 7.52 & 7.36 & 7.60 & 7.08 & \\
k1 & 1.65 & 1.76 & 1.86 & 1.84 & 1.81 & \\
k2 & 1.74 & 1.87 & 1.85 & 1.90 & 1.98 & \\
k3 & 1.93 & 1.93 & 1.89 & 1.80 & 1.88 & \\
k4 & 2.12 & 1.88 & 1.84 & 1.90 & 1.77 & \\
R & 0.48 & 0.18 & 0.06 & 0.10 & 0.21 & \\
\hline & & & & & & \\
\end{tabular}

Tab.3 Orthogonal analysis of variance table $(\alpha=0.05)$

\begin{tabular}{|c|c|c|c|c|c|c|}
\hline Factor & Squared & Freedom & F Ratio & $\mathrm{F}$ & Threshold & Significance \\
\hline A & 0.533 & 3 & 88.833 & & 9.280 & 米 \\
\hline B & 0.064 & 3 & 10.677 & & 9.280 & * \\
\hline $\mathrm{C}$ & 0.006 & 3 & 1.000 & & 9.280 & \\
\hline $\mathrm{D}$ & 0.032 & 3 & 5.333 & & 9.280 & \\
\hline $\mathrm{E}$ & 0.104 & 3 & 17.333 & & 9.280 & * \\
\hline Error & 0.01 & 3 & & & & \\
\hline
\end{tabular}

Tab. 2 and Tab. 3 can be seen in the five factors cellulase extracted barley Polysaccharide (hydrolysis time, hydrolysis temperature, enzyme dosage, $\mathrm{PH}$ value, the solid-liquid ratio), the maximum temperature hydrolysis, the solid-liquid than the least. The impact of the extraction rate of polysaccharides barley The order has to: hydrolysis temperature $>\mathrm{PH}$ value $>$ hydrolysis time $>$ enzyme $>$ solid-liquid ratio, namely $\mathrm{A}>\mathrm{E}>\mathrm{B}>\mathrm{D}>\mathrm{C}$, the experimental parameters on the basis of this, in order to obtain higher extraction rate, optimization of process parameters for each factor A4B3C3D4E2, namely hydrolysis temperature $55{ }^{\circ} \mathrm{C}$, hydrolysis time $120 \mathrm{~min}$, solid-liquid ratio 1:30, enzyme concentration $0.020 \mathrm{~g}, \mathrm{PH}$ value 4 , polysaccharide extracted under conditions rate of $2.23 \%$.

\section{Conclusion}

Effect of cellulase in barley polysaccharide extracted five factors, the maximum temperature hydrolysis, the solid-liquid ratio on the minimum. The impact of the extraction rate of polysaccharides barley The order has to: hydrolysis temperature $>\mathrm{PH}$ value $>$ hydrolysis time $>$ enzyme concentration $>$ solid-liquid ratio, based on the experimental parameters, in order to obtain a higher extraction rate, optimization of process parameters of various factors hydrolysis temperature is $55{ }^{\circ} \mathrm{C}$, hydrolysis time $120 \mathrm{~min}$, solid-liquid ratio of $1: 30$, the amount of enzyme $0.020 \mathrm{~g}$, PH value 4 , in this condition of polysaccharide extraction rate $2.23 \%$.

\section{Acknowledgements}

This work was financially Supported by the Dean Project of Guangxi Key Laboratory of Petrochemical Resource Processing and Process Intensification Technology(2012K09)

\section{References}

[1] CHANG H C, HUANG Y C, HUANG W C. Antiproliferative and chemo preventive effects of ad lay seed on lung cancer in vitro and in vivo[J]. Agric 
Food Chem, 2003, 51(12): 3656-3660.

[2] Yong-guang Bi, Yu-min Li. Study on the ultrasonic extraction of polysaccharides of barley rice[J]. Applied Mechanics and Materials, 2014, (513-517):332-335. 